\title{
Neue Kommunikationswege mit der „Ambulanz online“
}

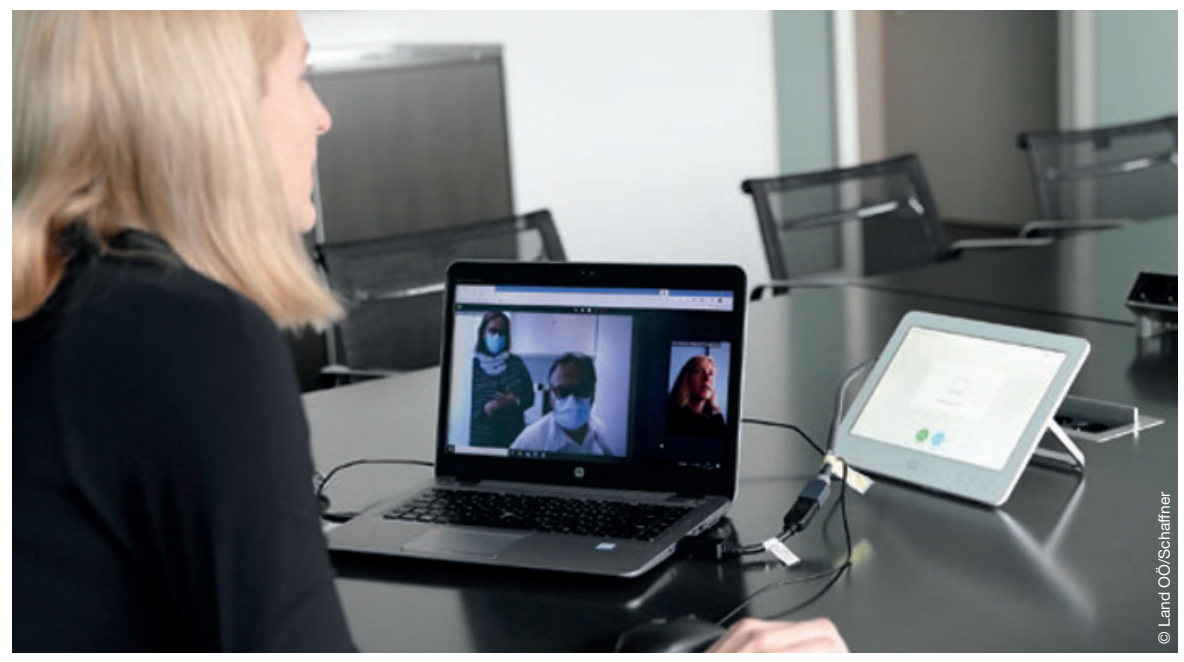

$\mathbf{N}$ eue Technologien haben den Gesundheitssektor schon immer stark beeinflusst und vorangebracht. Die Patientinnen und Patienten beschleunigen und fordern den Einsatz digitaler Technologien. Der Einsatz von digitalen Medien wird Einfluss auf die Qualität und die Attraktivität der Gesundheitsversorgung haben. Das Krankenhaus der Barmherzigen Schwestern Ried optimiert mit der Umsetzung der Ambulanz online die Versorgungskette für den Patienten und denkt die Digitalisierung konsequent aus der Sicht des Patienten.

\section{Patientenorientiert}

Die Sicherstellung einer exzellenten medizinischen Versorgung erfordert zunehmend zeitlich und räumlich flexible und damit auch digitale Lösungen in Ergänzung zum regulären Ambulanzangebot. Mit der Ambulanz online wird auf neue Kundenbedürfnisse eingegangen, um den digitalen Komfort im Alltag der medizinischen Behandlung einzubinden.

\section{Flexibel und sicher}

Die digitale Betreuung der Patienten kann - egal zu welcher Zeit und von welchem Ort aus (örtliche Distanz, eingeschränkte Mobilität, akute Situationen wie Pandemie...) - erfolgen. Patienten werden keinem Risiko einer Infektion ausgesetzt. Nicht-mobile Patienten, Krebspatienten und immunsupprimierten Patienten können dringend notwendige Beratungs- und Therapiegespräche mit ihrer medizinischfachlichen Bezugsperson im Krankenhaus führen.

\section{Praktikabel}

Als technische Voraussetzung sind für die Patienten lediglich ein PC, Laptop oder Mobiltelefon, eine stabile Internetverbindung und eine Kamera zur Videotelefonie erforderlich. Das neue virtuelle Angebot wurde in enger Abstimmung mit Rechts- und IT-Experten entwickelt. Beim Ambulanz online-Videochat wird nicht nur gesprochen; während man seinen Ge- sprächspartner sieht, kann auch die $\mathrm{Pa}$ tientenakte durch Teilen des Bildschirms gezeigt und somit beispielsweise Befundergebnisse gemeinsam mit den Patientinnen und Patienten besprochen werden. Im Unterschied zu gewöhnlichen Videokonferenz-Tools ist diese Anwendung maßgeschneidert und hoch sensibel im Umgang mit personenbezogenen Daten.

Mit dem Angebot der Ambulanz online wachsen die Individualisierung und das spezifische Eingehen auf Patientenpräferenzen und somit auch die Zufriedenheit der Patienten. Durch die Entlastung der Wartebereiche werden die Mitarbeitenden in den Ambulanzbereichen unterstützt. Die "Ambulanz online“ wird im Krankenhaus der Barmherzigen Schwestern Ried derzeit im Bereich der Inneren Medizin 1 eingesetzt, die Implementierung in weiteren Abteilungen ist geplant.

\section{Kontakt:}

Margit Matheis, BScN

Leitung Risikomanagement/Innovation

Krankenhaus der Barmherzigen Schwestern

Ried Betriebsgesellschaft m.b.H.,

Ein Unternehmen der Vinzenz Gruppe

margit.matheis@bhs.at

www.bhs-ried.at

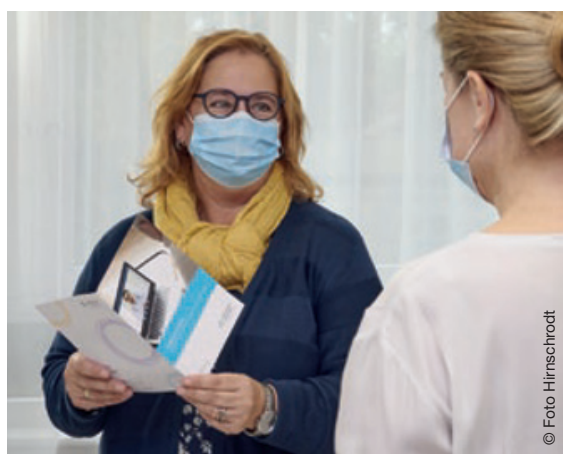

